\title{
Treatment of Middle Cerebral Artery Occlusion with Concomitant Partially Thrombosed Aneurysm: a Case Report
}

\author{
Michel Klapp Oliger $^{1} \cdot$ Till-Karsten Hauser $^{1} \cdot$ Franz-Josef Strauss $^{2,3} \cdot$ Ulrike Ernemann $^{1}$
}

Accepted: 5 November 2020 / Published online: 17 November 2020

(C) The Author(s) 2020

\begin{abstract}
A 68-year-old female with an acute ischemic stroke demanding emergency thrombectomy which was complicated by the presence of an intracranial aneurysm. Computer tomography revealed the occlusion of the right middle cerebral artery with a distal non-ruptured partially thrombosed aneurysm. The treatment consisted in the administration of intravenous recombinant tissue plasminogen activator and the removal of the proximal thrombus via direct aspiration in order to prevent perforation of the distal aneurysm. After the procedure, the clinical symptoms improved significantly indicated by the National Institutes of Health Stroke Scale (NIHSS). The aim of the present case report is, therefore, to suggest a clinical approach to help physicians in the decision-making process for early and safe revascularization in patients with ischemic stroke and intracranial aneurysms.
\end{abstract}

Keywords Aneurysm $\cdot$ MCA $\cdot$ Endovascular $\cdot$ Thrombosed $\cdot$ Thrombectomy $\cdot$ Direct aspiration

\section{Introduction}

The prompt restoration of the cerebral blood flow via reperfusion therapy is the most effective procedure to prevent tissue infarction due to ischemia. However, the efficacy of this procedure depends on the time of reperfusion as the benefits of reperfusion decrease over time. The first-line therapy in patients with acute ischemic stroke is intravenous alteplase (recombinant tissue plasminogen activator or tPA) which should be provided within the first $4.5 \mathrm{~h}$ following the onset of symptoms [1-3]. On the other hand, in patients with acute ischemic stroke but with a large artery occlusion in the anterior circulation, mechanical thrombectomy is indicated regardless of whether the patient had received intravenous alteplase for the same ischemic stroke event $[4,5]$.

Michel Klapp Oliger

michel.klapp-oliger@med.uni-tuebingen.de

1 Department of Diagnostic and Interventional Neuroradiology, University Hospital Tübingen, Tübingen, Germany

2 Clinic of Reconstructive Dentistry, Center of Dental Medicine, University of Zurich, Zurich, Switzerland

3 Department of Conservative Dentistry, School of Dentistry, University of Chile, Santiago, Chile
The acute occlusion of the middle cerebral artery in conjunction with a distal aneurysm of the same blood vessel is a rare event, but can be more common than we think, especially in older hypertensive women [6]. As a consequence, the available evidence is limited in terms of treatment approaches [6-8]. Here we present a successful interventional approach with mechanical thrombectomy of the middle cerebral artery in a patient with a concomitant distal aneurysm of the same vessel.

\section{Case Report}

A 68-year-old female was referred to our hospital approximately $2.5 \mathrm{~h}$ after acute onset of hemiparesis of the left side and aphasia. Clinical examination revealed severe dysarthria, sensomotoric hemiplegia of the left side and neglect to the left side (NIHSS score of 19). The patient was administered intravenous rtPA (IV-rtPA) about $2 \mathrm{~h}$ before the intervention started. CT scan of the brain demonstrated a dense aneurysm of the distal middle cerebral artery (MCA) on the right side (Fig. 1); intracranial CT angiography (CTA) demonstrated an occlusion of the right MCA of the M1-segment (Fig. 2). The aneurysm of the distal right MCA was non-perfused. After interdisciplinary discussion, the decision was made for an interventional approach with mechanical thrombectomy of the occluded M1-segment. Digital-subtraction angiography 


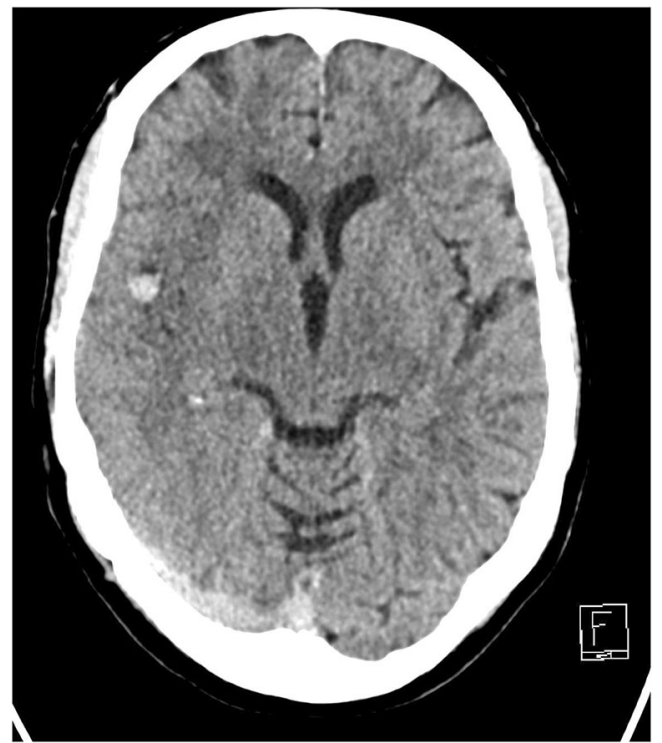

Fig. 1 Contrast head computed tomography demonstrated a dense aneurysm of the distal middle cerebral artery

(DSA) confirmed the right MCA occlusion. The aneurysm could not be seen in the DSA (Fig. 3).

\section{Interventional Procedure}

The intervention started approximately $5 \mathrm{~h}$ after onset of clinical symptoms and was performed under general anesthesia by one neuroradiologist. Digital-subtraction angiography confirmed the right MCA occlusion and verified the presence of a partially thrombosed aneurysm in the distal part of the right MCA. A retrograde perfusion of the distal aneurysm was

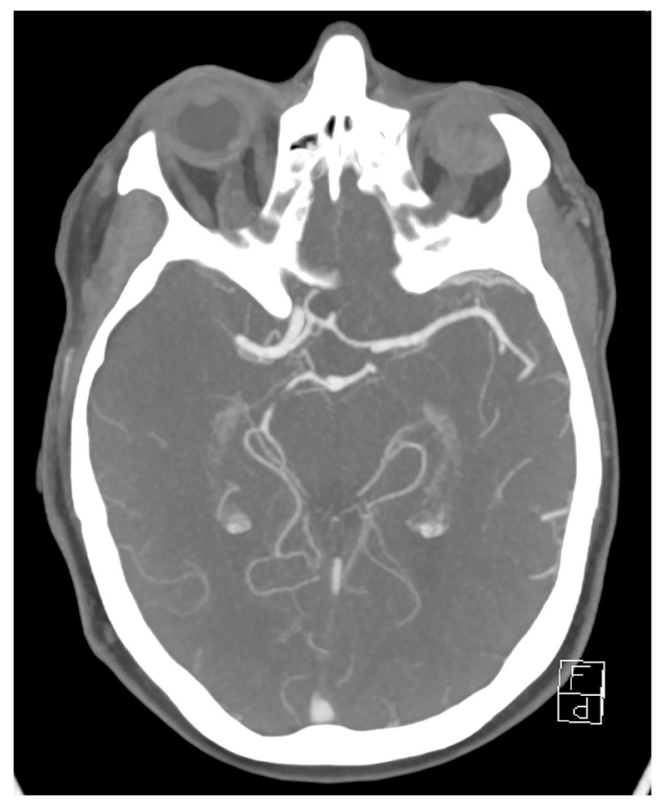

Fig. 2 Axial reconstructed image from the computed tomography confirms the right M1 segment middle cerebral artery occlusion

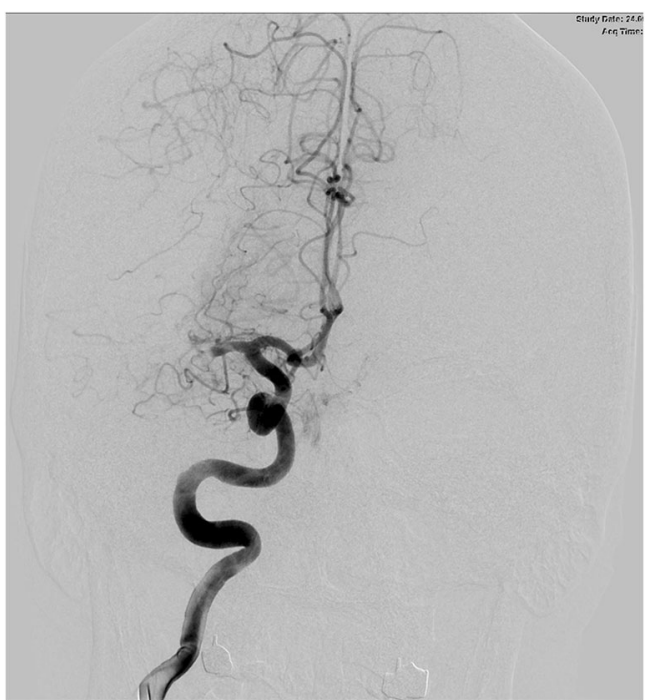

Fig. 3 Initial digital subtraction angiography image from the right internal carotid artery reveals an M1 middle cerebral artery occlusion

found. Because the presence of the clot was in the proximal right MCA and the aneurysm on the distal part, a decision was made to stay in the proximal part of the right MCA.

After insertion of a long $6 \mathrm{Fr}$ sheath (IVA6F80ST, BALT Extrusion) into the right common femoral artery, the sheath was advanced into the internal carotid artery with help of a 125-cm $5 \mathrm{Fr}$ vertebral catheter (Headhunter I Tempo Aqua, Cordis). Initial DSA series confirmed the occlusion of the middle cerebral artery in its M1 segment. Using a microcatheter (Vasco+21 MP, Balt Extrusion), a 6 Fr Sofia distal access catheter (MicroVention, Terumo) was placed in the cavernous segment of the ICA and advanced until the catheter tip made direct contact with the thrombus. As the aneurysm could not be visualized in the angiographic series, passing the thrombus with a microcatheter and deploying a stent retriever was judged to be too risky and a primary direct aspiration approach was opted for. A vacuum was applied to the distal access catheter by attaching a lockable $60 \mathrm{cc}$ syringe (Vaclok, Merit Medical Systems). After having aspirated the thrombus twice, first for 1-2 min and then for $5 \mathrm{~min}$, a complete reperfusion of the right MCA territory as well as the aneurysm was achieved (Fig. 4). No peripheral embolism was observed. The patient exhibited a significant improvement immediately after the procedure, with a NIHSS score of 10. A CT performed after the procedure showed an acute right putaminal infarction without signs of hemorrhagic transformation (Fig. 5). The patient experienced further neurological improvement during hospitalization, with mild residual expressive aphasia at discharge 9 days after stroke onset (NIHSS score $=3$ ). Anticoagulation with subcutaneous enoxaparin ( $80 \mathrm{mg}$ bid) was initiated at $72 \mathrm{~h}$ and was recommended to him until the subsequent aneurysm repair of the aneurysm (scheduled for 6 months later). 


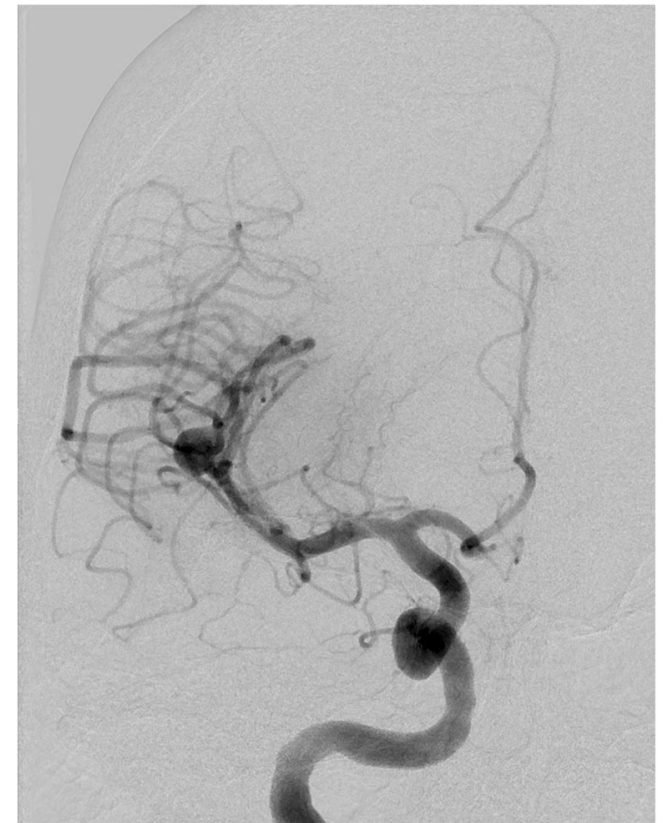

Fig. 4 Post-thrombectomy right internal carotid artery angiography documents excellent recanalization of the right middle cerebral artery and the distal aneurysm

\section{Discussion}

Here we show the safety and efficacy of a primary direct aspiration of a thrombus in a patient with a concomitant partially non-ruptured thrombosed aneurysm in the same blood vessel, in this case the right MCA. It should be noted though that the rupture of an aneurysm during thrombectomy is a lifethreatening complication, which should not deprive these patients of these procedures, but rather a particularly cautious

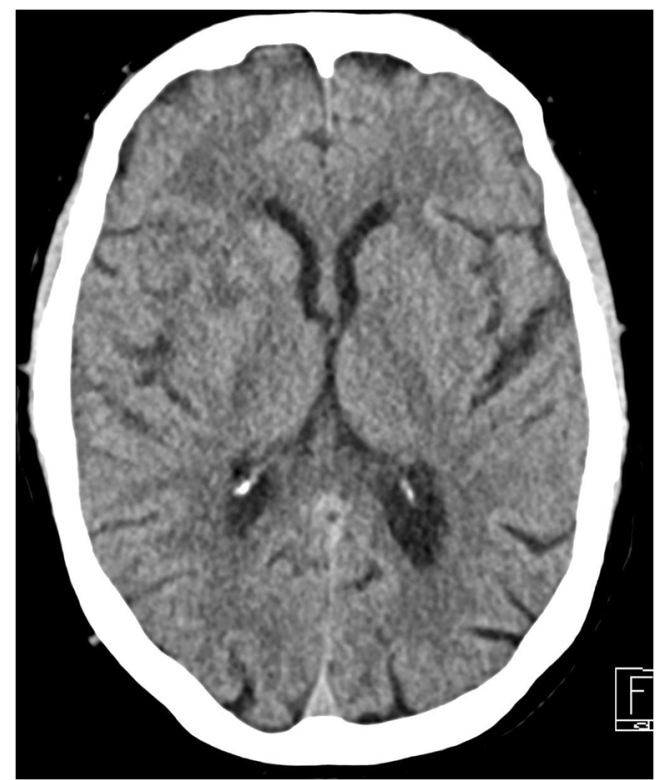

Fig. 5 Contrast head computed tomography demonstrated an acute right putaminal infarction without signs of hemorrhagic transformation approach may be justified [6-8]. The present case report supports this clinical maneuver in patients with large vessel occlusions thereby offering the possibility of a good functional recovery [3]. This procedure was performed to avoid the rupture of the aneurysm caused by the traditional thrombectomy with a stent retriever [6-8]. Even though aspiration thrombectomy might be safer than thrombectomy with stent retriever in an ipsilateral unruptured intracranial aneurysm, the evidence is lacking and there are no studies available comparing these two techniques. Although sometimes it is necessary to pass the thrombus with a microwire to achieve stability, good results can also be obtained with proximal aspiration of the occluded segment. The current case is among the few cases known of intracranial occlusion caused by a thrombus associated with a distal ipsilateral aneurysm in which a proximal thrombus aspiration was performed [6].

\section{Conclusions}

Both aspiration devices and stent retrievers are commonly used methods to achieve thrombectomy in acute ischemic stroke and they have a high success rate. Here we show that a mechanical thrombectomy of the middle cerebral artery with a concomitant distal aneurysm of the same vessel is feasible and with favorable clinical outcomes.

Funding Open Access funding enabled and organized by Projekt DEAL.

\section{Compliance with Ethical Standards}

Conflict of Interest The authors declare that they have no conflict of interest.

Ethical Approval All procedures performed in studies involving human participants were in accordance with the ethical standards of the institutional and/or national research committee and with the 1964 Helsinki Declaration and its later amendments or comparable ethical standards.

Informed Consent Informed consent was obtained from the participant included in the study.

Open Access This article is licensed under a Creative Commons Attribution 4.0 International License, which permits use, sharing, adaptation, distribution and reproduction in any medium or format, as long as you give appropriate credit to the original author(s) and the source, provide a link to the Creative Commons licence, and indicate if changes were made. The images or other third party material in this article are included in the article's Creative Commons licence, unless indicated otherwise in a credit line to the material. If material is not included in the article's Creative Commons licence and your intended use is not permitted by statutory regulation or exceeds the permitted use, you will need to obtain permission directly from the copyright holder. To view a copy of this licence, visit http://creativecommons.org/licenses/by/4.0/. 


\section{References}

1. Campbell BCV, Donnan GA, Lees KR, Hacke W, Khatri P, Hill $\mathrm{MD}$, et al. Endovascular stent thrombectomy: the new standard of care for large vessel ischaemic stroke. Lancet Neurol. 2015;14(8): 846-54.

2. Furlan AJ. Endovascular therapy for stroke - it's about time. N Engl J Med. 2015;372(24):2347-9.

3. Powers WJ, et al. 2018 guidelines for the early management of patients with acute ischemic stroke: a guideline for healthcare professionals from the American Heart Association/American Stroke Association. Stroke. 2018;49(3):e46-e110.

4. Chia NH, Leyden JM, Newbury J, Jannes J, Kleinig TJ. Determining the number of ischemic strokes potentially eligible for endovascular Thrombectomy: a population-based study. Stroke. 2016;47(5): 1377-80.
5. Cohen DL, et al. Around 9\% of patients with ischaemic stroke are suitable for thrombectomy. BMJ. 2015;351:h4607.

6. Zibold F, Kleine JF, Zimmer C, Poppert H, Boeckh-Behrens T. Aneurysms in the target vessels of stroke patients subjected to mechanical thrombectomy: prevalence and impact on treatment. J Neurointerv Surg. 2016;8(10):1016-20.

7. Singh J, Wolfe SQ. Stent retriever thrombectomy with aneurysm in target vessel: technical note. Interv Neuroradiol. 2016;22(5):544-7.

8. Torikoshi S, Akiyama Y. A concealed intracranial aneurysm detected after recanalization of an occluded vessel: a case report and literature review. Interv Neurol. 2016;4(3-4):90-5.

Publisher's Note Springer Nature remains neutral with regard to jurisdictional claims in published maps and institutional affiliations. 